\title{
Effect of Visual Field Constriction on Visual Search in Orderly Array and Random Array Cancellation Tasks
}

\author{
Ryotaro Saito1,2, Yoshifumi Ikeda ${ }^{3}$, Hideyuki Okuzumi' ${ }^{1}$, Iwao Kobayashi', \\ Mitsuru Kokubun1 \\ ${ }^{1}$ Tokyo Gakugei University, Koganei, Japan \\ ${ }^{2}$ Japan Society for the Promotion of Science, Tokyo, Japan \\ ${ }^{3}$ Joetsu University of Education, Joetsu, Japan \\ Email: r153002s@st.u-gakugei.ac.jp
}

Received 14 October 2015; accepted 10 November 2015; published 13 November 2015

Copyright (C) 2015 by authors and Scientific Research Publishing Inc.

This work is licensed under the Creative Commons Attribution International License (CC BY). http://creativecommons.org/licenses/by/4.0/

c) (i) Open Access

\begin{abstract}
The cancellation task is a visual search task requiring rapid and accurate placement of a mark through each occurrence of a specific target displayed in an array of distractors on a sheet of paper. This study of 30 university students was conducted to examine the effects of visual field constriction on visual search in the cancellation task. Participants administered the orderly array cancellation task and the random array cancellation task while their visual fields were unrestricted (full vision), or restricted to $5^{\circ}, 1^{\circ}$, or $15^{\circ}$ by tunnel vision simulation goggles. The number of errors, time to completion, and the performance score as an index of cancelling efficiency were calculated. Results showed that the time to completion and the performance score were significantly greater in the random array cancellation task than in the structured array cancellation task, and in the narrowest visual field condition $\left(5^{\circ}\right)$ than in other visual field conditions $\left(10^{\circ}, 1^{\circ}\right.$, and full vision). These results suggest that the visual search efficiency was degraded when the visual field was restricted to be less than $5^{\circ}$ in the orderly and random array cancellation tasks used for this study.
\end{abstract}

Keywords

Visual Scan, Visual Field, Tunnel Vision, Visual Angle, Planning

\section{Introduction}

Cancellation tasks are visual search tasks that are commonly used to assess attention or planning. A paper-and-

How to cite this paper: Saito, R., Ikeda, Y., Okuzumi, H., Kobayashi, I., \& Kokubun, M. (2015). Effect of Visual Field Constriction on Visual Search in Orderly Array and Random Array Cancellation Tasks. Psychology, 6, 1873-1878.

http://dx.doi.org/10.4236/psych.2015.614184 
pencil version of this task requires placement of a mark, as quickly and accurately as possible, through each occurrence of a specific target displayed in an array of distractors on a sheet of paper (e.g., Nakajima, Ikeda, \& Okuzumi, 2013). Performance is usually measured as visual search patterns, number/position of errors, time to complete the task, and the performance score (efficiency of cancelling) measured by accuracy and time.

Cancellation tasks have been used frequently over many years in the field of neuropsychology. These tasks have been used as sensitive measures for detecting patients with visual neglect (e.g., Weintraub \& Mesulum, 1988). Moreover, recent studies have used these tasks as an assessment of attentional ability in individuals with developmental disabilities such as dyslexia and attention deficit hyperactivity disorder (Huang \& Wang, 2009; Jones, Craver-Lemley, \& Barrett, 2008; Sandson, Bachna, \& Morin, 2000; Wang \& Huang, 2012).

Several factors influence cancellation task performance. First, performance is affected by the stimulus material (e.g., letters vs. shapes). Weintraub and Mesulum (1988) reported that more errors were observed when detecting shapes than when detecting letters. Secondly, the performance is affected by the target-to-distractor ratio. Studies reported lower accuracy and increased time as the proportion of distractors increased (Chatterjee, Mennemeier, \& Heilman, 1992; Geldmacher, 1996; Huang \& Wang, 2009; Kaplan, Verfaellie, Meadows, Caplan, Pessin, \& DeWitt, 1991). Third, performance is affected by a stimulus array (random vs. orderly). For healthy adults, Huang and Wang (2008) reported lower times in an orderly array, where stimuli are arranged in rows and columns, than in a random array, for which stimuli are not arranged in a structured manner.

Considering that the cancellation task is a visual search task, the visual field can be an important factor. Different parts of the visual field serve different functions during visual search. Whereas foveal vision is used to analyze any item currently in view, peripheral vision is used to select the next saccade target, i.e., planning saccades (Carpenter, 1991). Cornelissen, Bruin, and Kooijman (2005) investigated the influence of artificial peripheral scotomas on the orderly array visual search task, reporting increased search time for larger visual field defect. However, it remains unknown whether the artificial visual field defect also influences performance in the random array cancellation task, which is postulated to demand more planning to saccade than the orderly array cancellation task.

This study was conducted to investigate the effects of visual field constriction on visual search efficiency in orderly array and random array cancellation tasks in healthy adults while artificial visual field defects were created using tunnel vision simulation goggles. It was hypothesized that (a) cancelling efficiency would be higher in the orderly array than in the random array, (b) cancelling efficiency would decrease as visual fields narrow, and (c) decreases of cancelling efficiency with narrowed visual fields would be remarkable in a random array task that demands more planning because peripheral vision is used to select the next saccade target.

\section{Method}

\subsection{Participants}

This study examined 30 university students ( 15 men, 15 women; $\mathrm{M}=22.7 \mathrm{yr}, \mathrm{SD}=1.49$, range $=20$ - 26) who volunteered for the study after recruitment from a university in Tokyo, Japan. Participants self-reported that they were free of any physical illness, visual or motor problem, or perceptual or cognitive disorder. Only right-handed participants were retained as participants because the viewing angle for the stimuli varies with the dominant hand. In addition, all retained participants showed visual acuity of more than 1.0 on a near visual acuity test performed at $30 \mathrm{~cm}$ distance.

\subsection{Measure}

A paper-and-pencil version of the letter cancellation task was administered with two stimulus arrays and four visual fields: eight conditions. Across all conditions, a total of 374 stimulus letters were arranged in a standard white A4 paper (landscape, $21 \times 29 \mathrm{~cm}$ ): 60 targets (capital alphabet "A") and 314 distractors (capital alphabets "B" to "Z"). All targets and distractors were in black 11-point Arial font.

For stimulus arrays, two conditions were used. One was an orderly array in which stimuli were aligned in 17 rows $\times 22$ columns, with width spacing of $9 \mathrm{~mm}$ and vertical space of $6 \mathrm{~mm}$ open between stimuli. The other was a random array in which targets were placed at the same locations with the orderly array and distractors placed irregularly around the targets. Half of the targets (30 stimuli) of the arrays were arranged to be in the left panel, with the other half in the right panel. 
As for visual fields, four conditions were used: three constricted visuals fields of $5^{\circ}, 10^{\circ}$, and $15^{\circ}$, and a full visual field. Visual fields were constricted by tunnel vision simulation goggles (Takata Optical Co. Ltd.). Participants wore the goggles in the full visual field condition as well. During the tasks, the participant's left eye was covered so that the search the stimuli were viewed only from the right eye.

\subsection{Procedure}

Participants were tested individually at a desk in a well-lit, quiet room at a university. The test sheets were placed on the desk, centered approximately $30 \mathrm{~cm}$ from the edge and at the participant's midline. Each participant was instructed to use a pen with their dominant (right) hand to make a slash as quickly and accurately as possible through every occurrence of the target and not to slash through any occurrence of the distractor. Each participant was also instructed not to get closer to the sheet by bending the body or moving the sheet during the tasks. Each participant started the task time when an experimenter turned over the test sheet. Participants were instructed to put the pen down when they finished searching the targets. The order of the stimulus arrays was counterbalanced among the participants.

\subsection{Data Analysis}

Numbers of errors, numbers of correct responses, and time to complete a task were recorded. Items were counted as errors when a participant did not cancel a target (i.e., an omission error). No participant made a commission error, where a participant slashed a distractor. The experimenter used a stopwatch to time the interval between a participant's first mark and the placement of a pen on a desk signaling that the participant had finished searching targets.

Additionally, performance scores (PS) were calculated using the formula shown below:

PS = (number of correct responses/number of total items) $\times($ number of correct responses/time to complete a task)

PS was used frequently as an index of cancelling efficiency to evaluate both the accuracy and speed of the performance simultaneously (Brucki \& Nitrini, 2008; Geldmacher, 1996, 1998; Huang \& Wang, 2008, 2009). Higher PSs signify a larger number of correct responses per unit time. They reflect more efficient performance.

\subsection{Statistical Analysis}

To assess the time to complete a task and PS, a 2 (stimulus array; orderly, random) $\times 4$ (visual field; $5^{\circ}, 10^{\circ}, 15^{\circ}$, full) two-way analysis of variance (ANOVA) was conducted. For the number of errors where the data were not normally distributed, a Friedman test was conducted instead of ANOVA. For statistical analyses, software (SPSS Statistics ver. 22.0 for Windows; IBM Corp.) was used.

\subsection{Ethical Approval}

Our experimental protocol was administered in accordance with the guidelines of the Declaration of Helsinki and was approved by the institutional review board. Informed consent was obtained from all participants before the assessment session.

\section{Results}

\subsection{Number of Errors and Time to Complete a Task}

Table 1 presents means and standard deviations of number of errors and the time to complete a task for each condition. Overall, participants performed the task accurately. Then, because errors were not normally distributed, Friedman tests were conducted, respectively, for visual fields in the orderly array and the random array. Results showed no significant differences between visual fields (orderly array, $\chi^{2}=1.816 ; n$.s., random array, $\chi^{2}$ $=6.761, n . s$.$) . For the time to complete a task, two-way ANOVA showed significant effects of the stimulus ar-$ ray $\left(\mathrm{F}_{1,29}=14.983, p<.01\right.$, partial $\left.\eta^{2}=.3\right)$ and visual fields $\left(\mathrm{F}_{3,87}=23.298, p<.01\right.$, partial $\left.\eta^{2}=.445\right)$. Interaction between the stimulus array and visual fields was not significant $\left(\mathrm{F}_{3,87}=.526\right.$, n.s., partial $\left.\eta^{2}=.018\right)$. For visual fields, post hoc Bonferroni tests revealed significant differences between $5^{\circ}$ and others $(p<.01)$. 
Table 1. Means and standard deviations of the number of errors and task completion time.

\begin{tabular}{|c|c|c|c|c|c|}
\hline & & \multicolumn{4}{|c|}{ Visual field } \\
\hline & & Full & $15^{\circ}$ & $10^{\circ}$ & $5^{\circ}$ \\
\hline \multicolumn{6}{|l|}{ Orderly array } \\
\hline \multirow{2}{*}{ Number of errors } & $M$ & 1.00 & 1.00 & .80 & .70 \\
\hline & SD & 1.34 & 1.34 & 1.10 & 1.02 \\
\hline \multirow{2}{*}{ Time to complete a task } & $M$ & 64.35 & 66.83 & 67.01 & 79.69 \\
\hline & SD & 18.14 & 15.61 & 17.09 & 20.51 \\
\hline \multicolumn{6}{|l|}{ Random array } \\
\hline \multirow{2}{*}{ Number of errors } & $M$ & .97 & .93 & .57 & 1.40 \\
\hline & SD & 1.19 & 1.05 & .77 & 1.67 \\
\hline \multirow{2}{*}{ Time to complete a task } & $M$ & 69.38 & 71.53 & 73.95 & 87.24 \\
\hline & SD & 19.79 & 17.57 & 18.09 & 23.72 \\
\hline
\end{tabular}

\subsection{Performance Score}

Figure 1 depicts means and standard deviations of PS in each condition. Two-way ANOVA showed significant effects of the stimulus array $\left(\mathrm{F}_{1,29}=17.357, p<.01\right.$, partial $\left.\eta^{2}=.374\right)$ and visual fields $\left(\mathrm{F}_{3,87}=21.701, p<.01\right.$, partial $\left.\eta^{2}=.428\right)$. Interaction between the stimulus array and visual fields was not significant $\left(\mathrm{F}_{3,87}=.459\right.$, n.s., partial $\left.\eta^{2}=.016\right)$. Post hoc Bonferroni tests revealed significant differences between $5^{\circ}$ and others $(p<.01)$.

\section{Discussion}

This study was conducted to investigate whether visual field constriction affects visual search efficiency in orderly array and random array cancellation tasks in healthy adults.

Consistent with the hypothesis (a), results showed that the cancelling efficiency, measured as the time to complete the task and the performance score, is higher in the orderly array than in the random array across all visual field conditions. This result agrees with results of an earlier study (Huang \& Wang, 2008), which demonstrated that the time to complete the task was shorter and that the performance score for university students was higher for the orderly array task than for the random array task.

Inconsistent with hypothesis (b), results showed that the cancelling efficiency did not decrease linearly as the visual fields narrowed. The canceling efficiency was lower in the $5^{\circ}$ condition than in other conditions $\left(10^{\circ}, 15^{\circ}\right.$, and full), among which there were no further differences. Cornelissen et al. (2005) reported that the increased search time by peripheral visual defects was evidenced as an increase in the fixation duration and that this increase might not result from decreased recognition of stimuli because the foveal vision is not restricted except by the prolonged duration of saccade planning. Consequently, it is possible in this study that participants can use peripheral vision in visual field conditions, except for the $5^{\circ}$ condition. However, results of this study are not conclusive in this regard because the exact foveal and peripheral visual field sizes were not recorded.

Inconsistent with hypothesis (c), results showed no interactive effect between the stimulus arrays and the visual fields. It was expected that the cancelling efficiency reduction would be greater in random arrays because random arrays demand more planning for a search strategy and because peripheral vision is used to plan saccades. However, such is not the case. The reduction of cancelling efficiency in the $5^{\circ}$ condition is similar across stimulus arrays. It might be true that participant adults executed visual searches in an organized and systematic way because their planning ability was at a sufficiently mature level to create a search pattern, e.g., in a structured linear pattern in a left-to-right and top-to-bottom direction or because increasing visual fields result in reductions of return saccades and change in the saccade direction in order not to wander (Cornelissen et al., 2005).

There are many limitations in this study. First, the small sample limits its generalizability. Second, this study is not measure the personal range of foveal and peripheral visual field and the dominant eye. 


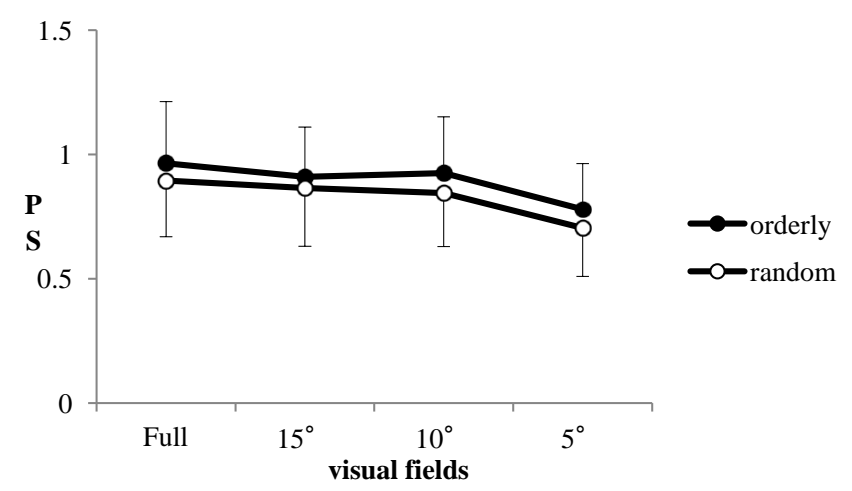

Figure 1. Means and standard deviations of the performance score.

Future studies are expected to examine the previously described possibility related to planning by individuals with immature planning, such as young children and people with intellectual and developmental disabilities.

\section{Conclusion}

Results of this study demonstrated that the cancelling efficiency decreased in orderly and random array cancellation tasks in healthy young adults when visual field was $5^{\circ}$. It is possible in this study that participants can use peripheral vision in visual field conditions, except for the $5^{\circ}$ condition.

\section{Acknowledgements}

This study was supported by Grants-in-Aid for JSPS Fellows Grant Number 15J11313 \& JSPS Kakenhi Grant Number 26381309.

\section{References}

Brucki, S. M. D., \& Nitrini, R. (2008). Cancellation Task in Very Low Educated People. Archives of Clinical Neuropsychology, 23, 139-147. http://dx.doi.org/10.1016/j.acn.2007.11.003

Carpenter, R. H. S. (1991). The Visual Origins of Ocular motility. Vision and Visual Function, 8, 1-10.

Chatterjee, A., Mennemeier, M., \& Heilman, K. M. (1992). A Stimulus-Response Relationship in Unilateral Neglect: The Power Function. Neuropsychologia, 30, 1101-1108. http://dx.doi.org/10.1016/0028-3932(92)90101-Q

Cornelissen, F. W., Bruin, K. J., \& Kooijman, A. C. (2005). The Influence of Artificial Scotomas on Eye Movements during Visual Search. Optometry \& Vision Science, 82, 27-35.

Geldmacher, D. S. (1996). Effects of Stimulus Number and Target-to-Distractor Ratio on the Performance of Random Array Letter Cancellation Tasks. Brain and Cognition, 32, 405-415. http://dx.doi.org/10.1006/brcg.1996.0073

Geldmacher, D. S. (1998). Stimulus Characteristics Determine Processing Approach on Random Array Letter-Cancellation Tasks. Brain and Cognition, 36, 346-354. http://dx.doi.org/10.1006/brcg.1997.0978

Huang, H.-C., \& Wang, T.-Y. (2008). Visualized Representation of Visual Search Patterns for a Visuospatial Attention Test. Behavior Research Methods, 40, 383-390. http://dx.doi.org/10.3758/BRM.40.2.383

Huang, H.-C., \& Wang, T.-Y. (2009). Stimulus Effects on Cancellation Task Performance in Children with and without Dyslexia. Behavior Research Methods, 41, 539-545. http://dx.doi.org/10.3758/BRM.41.2.539

Jones, K. E., Craver-Lemley, C., \& Barrett, A. M. (2008). Asymmetrical Visual-Spatial Attention in College Students Diagnosed with ADD/ADHD. Cognitive and Behavioral Neurology, 21, 176-178. http://dx.doi.org/10.1097/WNN.0b013e318185e6a9

Kaplan, R. F., Verfaellie, M., Meadows, M. E., Caplan, L. R., Pessin, M. S., \& Dewitt, L.D. (1991). Changing Attentional Demands in Left Hemispatial Neglect. Archives of Neurology, 48, 1263-1266.

http://dx.doi.org/10.1001/archneur.1991.00530240067023

Nakajima, Y., Ikeda, Y., \& Okuzumi, H. (2013). Target-to-Distractor Ratio Effects on Detection Time in the Orderly Array Shape Cancellation Task. Psychological Reports, 113, 353-361. http://dx.doi.org/10.2466/15.03.PR0.113x24z2

Sandson, T. A., Bachna, K. J., \& Morin, M. D. (2000). Right Hemisphere Dysfunction in ADHD Visual Hemispatial Inattention and Clinical Subtype. Journal of Learning Disabilities, 33, 83-90. http://dx.doi.org/10.1177/002221940003300111 
Wang, T. Y., \& Huang, H. C. (2012). The Performance on a Computerized Attention Assessment System between Children with and without Learning Disabilities. Procedia-Social and Behavioral Sciences, 64, 202-208. http://dx.doi.org/10.1016/j.sbspro.2012.11.024

Weintraub, S., \& Mesulam, M. M. (1988). Visual Hemispatial Inattention: Stimulus Parameters and Exploratory Strategies. Journal of Neurology, Neurosurgery, \& Psychiatry, 51, 1481-1488. http://dx.doi.org/10.1136/jnnp.51.12.1481 